\title{
Prevalence of Functional Gatrointestinal Diseases(FGID) and the Comparison of Psychological Symptoms according to the Presence of FGID in Psychiatric Out-patients at a University Hospital
}

Sang-Yeol Lee, Hye-Jin Lee Department of Psychiatry, Wonkwang University School of Medicine, Republic of Korea Department of Health, Wonkwang Grauduate School, Republic of Korea

\begin{abstract}
AIM: This study investigated the prevalence of functional gastrointestinal disease(FGID) and comparison of psychological symptoms according to the presence of FGID in psychiatric outpatients at a University Hospital.

METHODS: The study was conducted with a total of 170 outpatients visiting the Department of Psychiatry at a University Hospital. After excluding 26 patients due to unreliable responses, the remaining 144 patients were included in the data analysis. FGID was identified in accordance with the Rome III questionnaire - Korean version. Demographic factors were recorded, and psychosocial factors were evaluated using the Hospital Anxiety Depression Scale (HADS), Patient Health Questionnaire-15 (PHQ-15), Childhood Trauma QuestionnaireKorean (CTQ-K), and State-Trait Anger Expression Inventory (STAXI). Chi-squared test, one-way ANOVA, Student's $t$-test and the Pearson's correlation coefficient was used as statistical analysis methods.
\end{abstract}

CONCLUSIONS: This study found that patients with psychiatric disorders had a high prevalence of FGIDs, and psychiatric outpatients with FGIDs showed higher psychopathology. These results suggest that FGIDs could be evaluated and managed for the treatment of psychiatric out-patients.

Table 3. Comparison of anxiety, depressive symptom, somatic symptoms and childhood trauma among FGID subtypes

\begin{tabular}{|c|c|c|c|c|c|c|c|c|}
\hline & \multicolumn{2}{|c|}{ IBS } & \multicolumn{2}{|c|}{ FD } & \multicolumn{2}{|c|}{$\mathrm{FC}$} & \multicolumn{2}{|c|}{ NERD } \\
\hline & $\begin{array}{l}\text { Negative } \\
(n=120)\end{array}$ & $\begin{array}{l}\text { Positive } \\
(n=24)\end{array}$ & $\begin{array}{l}\text { Negative } \\
(n=115)\end{array}$ & $\begin{array}{l}\text { Positive } \\
(\mathrm{n}=29)\end{array}$ & $\begin{array}{l}\text { Negative } \\
(n=118)\end{array}$ & $\begin{array}{l}\text { Positive } \\
(n=26)\end{array}$ & $\begin{array}{l}\text { Negative } \\
(n=80)\end{array}$ & $\begin{array}{l}\text { Positive } \\
(n=64)\end{array}$ \\
\hline \multirow[t]{2}{*}{ Anxiety (HADS) } & $7.54 \pm 5.83$ & $11.71 \pm 6.03$ & $7.09 \pm 5.56$ & $12.79 \pm 5.81$ & $8.11 \pm 5.99$ & $8.81 \pm 6.35$ & $6.41 \pm 5.47$ & $10.52 \pm 5.99$ \\
\hline & \multicolumn{2}{|c|}{$t=-3.106, p=0.002$} & \multicolumn{2}{|c|}{$t=-4.893, p<0.001$} & \multicolumn{2}{|c|}{$t=-0.531, p=0.596$} & \multicolumn{2}{|c|}{$t=-4.286, p<0.001$} \\
\hline \multirow{3}{*}{$\begin{array}{l}\text { Depressive symptom } \\
\text { (HADS) }\end{array}$} & $8.69 \pm 5.34$ & $11.21 \pm 5.39$ & $8.36 \pm 5.21$ & $12.10 \pm 5.25$ & $8.79 \pm 5.17$ & $10.58 \pm 6.27$ & \multirow{2}{*}{\multicolumn{2}{|c|}{$\begin{array}{c}7.79 \pm 5.18 \quad 10.77 \pm 5.27 \\
t=-3.402, p<0.001\end{array}$}} \\
\hline & \multicolumn{2}{|c|}{$t=-2.105, p=0.037$} & \multicolumn{2}{|c|}{$t=-3.459, p<0.001$} & \multicolumn{2}{|c|}{$t=-1.534, p=0.127$} & & \\
\hline & $\begin{array}{l}7.21 \pm 6.13 \\
t=-3.565\end{array}$ & $\begin{array}{l}12.08 \pm 6.01 \\
p<0.001\end{array}$ & $\begin{array}{l}6.26 \pm 5.23 \\
t=-7.906\end{array}$ & $\begin{array}{l}15.00 \pm 5.68 \\
p<0.001\end{array}$ & $\begin{array}{r}7.85 \pm 6.45 \\
t=-0.695\end{array}$ & $\begin{array}{l}8.81 \pm 6.01 \\
p=0.488\end{array}$ & \multicolumn{2}{|c|}{$\begin{array}{c}5.10 \pm 4.95 \quad 11.67 \pm 6.06 \\
t=-7.162, p<0.001\end{array}$} \\
\hline CTQ-K & \multicolumn{8}{|c|}{$43.20 \pm 13.03 \quad 43.79 \pm 18.6943 .20 \pm 12.86$} \\
\hline \multicolumn{9}{|c|}{$\begin{array}{l}\text { N: Numer, FGID : Functional gastrointestinal disorder, NERD : Nonerosive reflux disease, FD : Functional dyspepsia, FC : Function- } \\
\text { al constipation, BS : Imitable bowel syndrome, HADS : Hospital Anxiety Depression Scale, PHQ-15 : Patient Health Questionnire-15, } \\
\text { CTQ-K: Childhood Trauma Questionnaire-Korean }\end{array}$} \\
\hline \multicolumn{9}{|c|}{ Table 4. Comparison of trait anger, state anger, anger-in, anger-out and anger control among the FGID subtypes } \\
\hline & \multicolumn{2}{|c|}{ IBS } & \multicolumn{2}{|c|}{$F D$} & \multicolumn{2}{|c|}{$\mathrm{FC}$} & \multicolumn{2}{|c|}{ NERD } \\
\hline & $\begin{array}{l}\text { Negative } \\
(n=120)\end{array}$ & $\begin{array}{l}\text { Positive } \\
(n=24)\end{array}$ & $\begin{array}{l}\text { Negative } \\
(n=115)\end{array}$ & $\begin{array}{l}\text { Positive } \\
(n=29)\end{array}$ & $\begin{array}{l}\text { Negative } \\
(n=118)\end{array}$ & $\begin{array}{l}\text { Positive } \\
(n=26)\end{array}$ & $\begin{array}{l}\text { Negative } \\
(n=80)\end{array}$ & $\begin{array}{l}\text { Positive } \\
(n=64)\end{array}$ \\
\hline . & $\begin{array}{r}22.08 \pm 6.78 \\
t=-3.683,\end{array}$ & $\begin{array}{l}28.04 \pm 9.24 \\
p<0.001\end{array}$ & $\begin{array}{r}21.38 \pm 6.68 \\
t=-4.148\end{array}$ & $\begin{array}{l}28.00 \pm 8.79 \\
, p<0.001\end{array}$ & $\begin{array}{r}22.98 \pm 7.81 \\
t=-0.315\end{array}$ & $\begin{array}{l}23.50 \pm 6.33 \\
, p=0.753\end{array}$ & $\begin{array}{r}21.44 \pm 6.36 \\
t=-2.994\end{array}$ & $\begin{array}{l}25.13 \pm 8.41 \\
4, p=0.003\end{array}$ \\
\hline i & $\begin{array}{r}18.47 \pm 7.89 \\
t=-0.071\end{array}$ & $\begin{array}{l}16.74 \pm 5.30 \\
p=0.928\end{array}$ & $\begin{array}{r}17.79 \pm 7.54 \\
t=-2.181\end{array}$ & $\begin{array}{l}21.28 \pm 8.26 \\
p=0.031\end{array}$ & $\begin{array}{r}18.43 \pm 7.94 \\
t=-0.199\end{array}$ & $\begin{array}{l}18.77 \pm 7.18 \\
9, p=0.843\end{array}$ & $\begin{array}{r}17.20 \pm 7.88 \\
t=-2.259\end{array}$ & $\begin{array}{l}20.11 \pm 7.41 \\
9, p=0.025\end{array}$ \\
\hline Anger-in & $\begin{array}{r}16.74 \pm 5.30 \\
t=-2.463\end{array}$ & $\begin{array}{l}19.67 \pm 5.37 \\
p=0.015\end{array}$ & $\begin{array}{r}16.63 \pm 5.22 \\
t=-2.684\end{array}$ & $\begin{array}{l}19.59 \pm 5.56 \\
\mathrm{p}=0.008\end{array}$ & $\begin{array}{r}17.22 \pm 5.50 \\
t=-0.042\end{array}$ & $\begin{array}{l}17.27 \pm 5.05 \\
2, p=0.967\end{array}$ & $\begin{array}{r}16.14 \pm 5.39 \\
t=-2.772\end{array}$ & $\begin{aligned} 18.59 \pm 5.13 \\
2, p=0.006\end{aligned}$ \\
\hline Anger-out & $\begin{array}{r}15.87 \pm 4.86 \\
t=-2.355\end{array}$ & $\begin{array}{l}18.46 \pm 5.19 \\
p=0.020\end{array}$ & $\begin{array}{r}15.69 \pm 4.85 \\
t=-3.005\end{array}$ & $\begin{array}{l}18.72 \pm 4.91 \\
p=0.003\end{array}$ & $\begin{array}{r}16.22 \pm 5.01 \\
t=-0.399\end{array}$ & $\begin{array}{l}16.65 \pm 5.08 \\
, p=0.690\end{array}$ & $\begin{array}{r}15.23 \pm 4.78 \\
t=-2.958\end{array}$ & $\begin{array}{l}17.64 \pm 4.96 \\
8, p=0.004\end{array}$ \\
\hline Anger-control & $\begin{array}{r}19.57 \pm 5.71 \\
t=-0.923\end{array}$ & $\begin{array}{l}20.75 \pm 5.89 \\
p=0.358\end{array}$ & $\begin{array}{r}19.65 \pm 5.81 \\
t=-0.464\end{array}$ & $\begin{array}{l}20.21 \pm 5.47 \\
p=0.643\end{array}$ & $\begin{array}{r}19.85 \pm 5.71 \\
t=0.372\end{array}$ & $\begin{array}{l}19.38 \pm 5.89 \\
p=0.711\end{array}$ & $\begin{array}{r}19.39 \pm 5.91 \\
t=0.880\end{array}$ & $\begin{array}{l}20.23 \pm 5.52 \\
, p=0.380\end{array}$ \\
\hline
\end{tabular}

Table 1, Demographic and clinical characteristics of paricipants

\begin{tabular}{|c|c|c|c|c|c|}
\hline & & FGD-positive $(n=63)$ & FGI-negative $(n=81)$ & $x^{2} / 4$ & $p$ \\
\hline \multirow[t]{2}{*}{$\operatorname{Sex}, n(\mathbb{R})$} & Male & $32(50,8)$ & $45(55.6)$ & 0.323 & 0.570 \\
\hline & Female & $31(49,2)$ & $36(44,4)$ & & \\
\hline Age & $M \pm S D$ & $43,22 \pm 15,13$ & $45.44 \pm 14.52$ & 0.890 & 0.375 \\
\hline \multirow[t]{5}{*}{ Marital status, $n\left(\%_{0}\right)$} & Unmaried & $26(41.3)$ & $37(45.7)$ & 1.460 & 0.834 \\
\hline & Maried & $24(38.1)$ & $30(37,0)$ & & \\
\hline & Separated & $2(3.2)$ & $2(2,5)$ & & \\
\hline & Divorced & $6(9,5)$ & $9(11.1)$ & & \\
\hline & Bereaved & $5(7,9)$ & $3(3.7)$ & & \\
\hline \multirow[t]{5}{*}{ Education, years (\%) } & None & $1(1.6)$ & $0(0,0)$ & 10.139 & 0.017 \\
\hline & $<6$ & $8(12,7)$ & $10(12,3)$ & & \\
\hline & $6-9$ & $28(44.4)$ & $32(39.5)$ & & \\
\hline & $10-12$ & $20(31,7)$ & $30(37,0)$ & & \\
\hline & $>12$ & $6(9,6)$ & $9(11.2)$ & & \\
\hline \multirow[t]{5}{*}{ Diognosis, $n\left(\mathbb{F}_{0}\right)$} & Schizophrenia & $36(57.1)$ & $30(37,0)$ & 6.717 & 0.152 \\
\hline & Mood disorder & $18(28.6)$ & $32(39.5)$ & & \\
\hline & Anxiety disorder & $2(3.2)$ & $5(6.2)$ & & \\
\hline & Cognitive disorder & $4(6.3)$ & $5(6.2)$ & & \\
\hline & AUD & $3(4,8)$ & $9(11.1)$ & & \\
\hline \multirow[t]{2}{*}{ Smoking, n $(\%)$} & Yes & $19(30.2)$ & $25(30.9)$ & 0.008 & 0.927 \\
\hline & No & $44(69,8)$ & $56(69.1)$ & & \\
\hline \multirow[t]{2}{*}{ Alcohol, $n\left(\%_{0}\right)$} & Yes & $29(46,0)$ & $39(48.1)$ & 0.814 & 0.606 \\
\hline & No & $34(54,0)$ & $42(51.9)$ & & \\
\hline \multirow[t]{2}{*}{ Antiocidic ogent, $n\left(\%_{0}\right)$} & Yes & $6(9,5)$ & $13(16.0)$ & 1.318 & 0.324 \\
\hline & No & $57(90.5)$ & $68(84,0)$ & & \\
\hline \multirow[t]{2}{*}{ Chronic diseose, $n(\%)$} & Yes & $23(36.5)$ & $27(33,3)$ & 0.158 & 0.691 \\
\hline & No & $40(63.5)$ & $54(66.7)$ & & \\
\hline
\end{tabular}

FGD: Functional gastrointestinal disorder, N : Number, M: Mean, SD : Standard deviation, AUD : Alcohol use disorder

Table 2. Comparison of psychiatric disorder by FGID group

\begin{tabular}{|c|c|c|c|c|c|c|}
\hline & & $\begin{array}{c}\text { Schizophrenia } \\
n(\%)\end{array}$ & $\begin{array}{c}\text { Mood disorder } \\
n(\%)\end{array}$ & $\begin{array}{c}\text { Anxiety disorder } \\
n(\%)\end{array}$ & $\begin{array}{c}\text { Cognitive disorder } \\
n(\%)\end{array}$ & $\begin{array}{c}\text { Alcohol use disorder } \\
n(\%)\end{array}$ \\
\hline \multirow[t]{3}{*}{$\operatorname{IBS}(n=24)$} & Negative & $60(90.9)$ & $39(78.0)$ & $4(57.1)$ & $9(100)$ & $8(66.7)$ \\
\hline & Positive & $6(9.1)$ & $11(22.0)$ & $3(42.9)$ & $0(0.0)$ & $4(33.3)$ \\
\hline & & \multicolumn{5}{|c|}{$x^{2}=11.408, p=0.022$} \\
\hline \multirow[t]{3}{*}{$F D(n=29)$} & Negative & $58(87.9)$ & $35(70.0)$ & $5(71.4)$ & $7(77.8)$ & $10(83.3)$ \\
\hline & Positive & $8(12.1)$ & $15(30.0)$ & $2(28.6)$ & $2(22.2)$ & $2(16.7)$ \\
\hline & & \multicolumn{5}{|c|}{$x^{2}=6.085, p=0.193$} \\
\hline \multirow[t]{3}{*}{$F C(n=26)$} & Negative & $55(83.3)$ & $41(82.0)$ & $7(100)$ & $6(66.7)$ & $9(75.0)$ \\
\hline & Positive & $11(16.7)$ & $9(18.0)$ & $0(0.0)$ & $3(33,3)$ & $3(25.0)$ \\
\hline & & \multicolumn{5}{|c|}{$x^{2}=3.439, p=0.487$} \\
\hline \multirow[t]{3}{*}{ NERD $(n=64)$} & Negative & $43(65.2)$ & $23(46.0)$ & $2(28.6)$ & $6(66.7)$ & $6(50.0)$ \\
\hline & Positive & $23(34.8)$ & $27(54,0)$ & $5(71.4)$ & $3(33.3)$ & $6(50.0)$ \\
\hline & & \multicolumn{5}{|c|}{$x^{2}=6.975, p=0.137$} \\
\hline
\end{tabular}

N: Numer, FGID : Functional gastrointestinal disorder, NERD : Nonerosive reflux disease, FD : Functional dyspepsia, FC : Functional constipation, IBS: Imitable bowel syndrome 